\title{
Experiencia fonoaudiológica con niños y niñas para la promoción de derechos: análisis desde el enfoque comunitario
}

Nelson Muñoz Lizana ${ }^{\mathbf{a}, *}$, Christian Peñaloza Castillo ${ }^{\mathbf{a}}$

a Departamento de Fonoaudiología, Universidad de Chile, Chile

\section{RESUMEN}

El artículo analiza, desde los planteamientos del enfoque comunitario, una experiencia fonoaudiológica para la promoción de derechos de niños y niñas, efectuada en una organización social, con participación de un grupo de estudiantes de la carrera de Fonoaudiología de la Universidad de Chile en el año 2013. En los abordajes comunitarios, la comunidad construye conocimientos y realiza acciones para lograr el fortalecimiento de sus relaciones, bienestar social, justicia, autonomía y emancipación. El análisis de la experiencia se realiza a partir de una selección de las bitácoras de campo del equipo de estudiantes participantes, en base a los principios y dimensiones del paradigma de la construcción y transformación crítica de Maritza Montero (2004). Los resultados se dividen en tres momentos (inicio, transición y reconfiguración del trabajo), los cuales dan cuenta del grado de complejidad del proceso y los alcances obtenidos en base a las dimensiones: ontológica, epistemológica, metodológica, ética y política del enfoque comunitario. La discusión enfatiza la importancia de la reflexión crítica sobre los fundamentos y las prácticas de la Fonoaudiología en el abordaje de la comunicación y el desarrollo humano, para aportar a la justicia social desde el enfoque comunitario.

\section{Speech-language therapy experience with children for the promotion of rights: analysis from a community-based approach}

\begin{abstract}
This paper analyzes, from a community-based approach, a speech, language and hearing sciences therapy experience for the promotion of children's rights, carried out in a social organization, with the participation of a group of speech, language and hearing sciences therapy undergraduate students at the Universidad de Chile in 2013. In community approaches, all community members build knowledge and take actions to build stronger social relationships and welfare, justice, autonomy and emancipation. The analysis of this experience was conducted by selecting sections from the fieldwork diaries of the team of participating students, based on the principles and dimensions of the paradigm of construction and critical transformation of Maritza Montero (2004). The results were divided into three moments (beginning, transition and reset of the work), which account for the level of complexity of the process and the goals achieved based on the ontological, epistemological, methodological, ethical and political dimensions of the community-based approach. The discussion emphasizes the importance of critical reflection on the foundations and practices of speech, language and hearing sciences therapy in the approach to communication and human development, to contribute to social justice from a community perspective.
\end{abstract}

\section{Palabras clave:}

Fonoaudiología;

Fonoaudiología

Comunitaria;

Comunicación; Promoción de derechos; Enfoque comunitario

\section{Keywords:} Speech-language pathology; Communitybased speech-language therapy; Communication; Promotion of rights; Community-based approach 


\section{INTRODUCCIÓN}

América Latina, junto con los territorios que abarcan el sur global, enfrentan una serie de injusticias sociales, políticas y culturales de naturaleza multifacética y estructural, producto del capitalismo y el colonialismo occidental hegemónico a nivel mundial (Santos, 2011). La pobreza, la exacerbación de los conflictos nacionales y étnicos, la violencia de género, la violación sistemática de Derechos Humanos y la degradación medioambiental (Escobar et al., 2020) son algunas de las expresiones de desigualdad social que se producen y perpetúan en este orden mundial. A esto se suma la injusticia epistémica o cognitiva imperante en la academia, que tiende a reconocer únicamente como válidos los conocimiento y las prácticas profesionales que emergen dentro de los márgenes del positivismo, euro y androcéntricos (Harding, 1996; Santos, 2011).

Frente a este escenario, se vuelve urgente impulsar acciones que, desde distintos niveles y espacios, contribuyan a la eliminación de cualquier opresión, con el fin de transformar y construir sociedades más justas e inclusivas, respetuosas de la diversidad cultural, en el marco de los Derechos Humanos. Dentro de las apuestas transformadoras se encuentra el enfoque comunitario, alternativa epistémica, ética y política que cuenta con un importante desarrollo en Latinoamérica, tanto desde la psicología (Alfaro, 2000; Flores, 2012; Martínez, 2006; Montero, 2004; Rozas, 2015), como desde el área de salud mental (Cohen \& Natella, 2013; Marconi, 1972) y de rehabilitación (García-Ruiz, 2014; García-Ruiz et al., 2019).

La propuesta comunitaria se sustenta en la concepción de sujetos y comunidades como unidades sociales históricas, en constante transformación, que en su interrelación entre los integrantes que la componen y con otros colectivos, generan un sentido de pertenencia e identidad (Krause, 2001; Montero, 2004; Rozas, 2015). Las acciones comunitarias buscan que las comunidades fortalezcan sus capacidades, sus procesos de comunicación, de construcción colectiva y que reflexionen de manera crítica sobre las condiciones de opresión que las excluyen, estigmatizan, explotan e inferiorizan, con el fin de generar conocimiento situado y acciones para la emancipación.

Montero (2004) sitúa al abordaje comunitario en el paradigma de la construcción y transformación crítica, en el cual la relación intersubjetiva es el ámbito fundamental de la construcción de las personas, del conocimiento y de la praxis comunitaria. Por tanto, la comunidad tiene un rol participativo activo en la generación y organización del conocimiento. Se rompe, así, la concepción de trabajar con individuos aislados, no situados en una historia ni en redes de interacción y poder.

La finalidad de cualquier abordaje comunitario es la transformación de las comunidades, en pos de un mayor bienestar, justicia, autonomía y calidad en la participación (Flores, 2012; Prilleltensky, 2004; Rozas, 2015). Las acciones comunitarias, por lo tanto, deben evidenciar un aporte claro en ese sentido. Para ello, la reflexión crítica es parte del compromiso por el bienestar y la transformación de las comunidades, en que las relaciones de poder están siempre presentes. Actuar con el fin de promover el bienestar personal, relacional y colectivo, incrementando la acción, la participación y el compromiso político de los actores sociales, les otorga a los procesos de generación de conocimiento la validez psicopolítica (Prilleltensky, 2004) a la cual deberían aspirar todas las acciones definidas como comunitarias.

Montero (2004) propone que lo anterior obliga a reflexionar sobre la actuación profesional con comunidades desde un conjunto de dimensiones interrelacionadas e inherentes a cualquier intervención comunitaria: la ontológica, la epistemológica, la metodológica, la ética y la política. Estas dos últimas se incluyen en contraposición a modelos de producción de conocimiento que las invisibiliza, con la falsa pretensión de una construcción de conocimiento desideologizado, universal, apolítico, ahistórico y neutral, que caracteriza a la ciencia (Martínez Hernáez, 2011). En el siguiente cuadro, se sintetiza lo esencial de cada una de las dimensiones, según la caracterización hecha por Montero. 
Cuadro 1. Descripción de las dimensiones paradigmáticas de un enfoque comunitario.

\begin{tabular}{|c|c|}
\hline Dimensión & Características \\
\hline Ontológica & $\begin{array}{l}\text { El sujeto no es un ser individual, aislado, sino alguien que construye realidad y que protagoniza la vida cotidiana en relaciones con } \\
\text { otros. Toda consideración pasiva de la comunidad debe ser desechada y, por lo tanto, sus miembros tienen el derecho de tomar } \\
\text { decisiones sobre aquellos asuntos que les conciernen. }\end{array}$ \\
\hline Epistemológica & $\begin{array}{l}\text { La producción de conocimiento surge en la relación entre sujetos y no entre sujetos conocedores y sujeto objetos. Los participantes } \\
\text { construyen una realidad a partir de la relación, lo que a su vez los transforma, los limita y los impulsa, en un continuo proceso } \\
\text { dinámico. La realidad es inherente a los sujetos que la construyen cada día, activa y simbólicamente, en un constante movimiento } \\
\text { que no sólo es dialéctico, sino que también puede ser analéctico. A su vez, los sujetos están en la realidad, son parte de ella, y no } \\
\text { es posible separarles.. }\end{array}$ \\
\hline Metodológica & $\begin{array}{l}\text { Investigación-acción en su expresión participativa (IAP). Exige la necesidad de generar métodos que se transformen al mismo } \\
\text { ritmo que cambian las comunidades. Métodos cuya característica fundamental sea la capacidad de cambiar según los cambios del } \\
\text { problema que estudia, de tal manera que se generen construcciones en una acción crítica y reflexiva de carácter colectivo. }\end{array}$ \\
\hline Ética & $\begin{array}{l}\text { Tiene como objetivo principal la relación con el Otro en términos de igualdad y respeto, incluyendo la responsabilidad que cada } \\
\text { uno tiene respecto del Otro. Significa reconocer la existencia independiente de la comunidad como forma de grupo y de sus } \\
\text { miembros en su singularidad, en su carácter de dueños de una historia construida por ellos, anteriores a la intervención comunitaria } \\
\text { y posteriores a ella. Una ética fundamentada en la relación supone considerar el interés común por encima del bienestar individual. } \\
\text { La relación es siempre dialógica, lo que significa que las relaciones humanas tienen que ser abiertas a una multiplicidad de voces. }\end{array}$ \\
\hline Política & $\begin{array}{l}\text { Se propone un espacio de acción transformadora. El paradigma del enfoque comunitario propone una participación cuyo carácter } \\
\text { político se muestra en la función desalienante, movilizadora de la conciencia y socializadora, que puede tener la praxis llevada a } \\
\text { cabo. Asimismo, la construcción de conocimiento y el respeto a la diversidad tienen consecuencias políticas, liberadoras. }\end{array}$ \\
\hline
\end{tabular}

Fuente: Montero (2004).

En el contexto chileno, existen diversas experiencias de Fonoaudiología que han explorado una interacción en comunidades, principalmente en dispositivos de salud. Dichas actuaciones se rigen por un enfoque a lo sumo biopsicosocial, con escasas acciones dirigidas al fortalecimiento de las comunidades (Silva et al., 2018; Tapia et al., 2016). Por tanto, los principios del enfoque comunitario se mantienen ausentes en dichas prácticas. Desde ese punto de vista, este artículo se propone analizar de manera crítica una experiencia fonoaudiológica con niños y niñas en una organización social, con la participación de un grupo de estudiantes de la carrera de Fonoaudiología de la Universidad de Chile, en el marco del desarrollo de su tesis de licenciatura (Calixto et al., 2013). Se aborda desde los fundamentos paradigmáticos del enfoque comunitario, para relevar los desafíos, las contradicciones, y las oportunidades que representan para la Fonoaudiología una experiencia que intente ser genuinamente comunitaria.

\section{DESCRIPCIÓN DE LA EXPERIENCIA}

La experiencia se realizó entre los meses de marzo y noviembre del año 2013, en las instalaciones de la Corporación Colectivo Sin
Fronteras, de la comuna de Independencia, Santiago de Chile. El Colectivo Sin Fronteras, creado en el año 2003, tiene por objetivo "promover la integración social y la interculturalidad, así como el respeto y ejercicio de derechos de salud, no discriminación, protección contra malos tratos, nivel de vida adecuado y educación de niños y niñas migrantes latinoamericanos y sus familias, impulsando condiciones que favorezcan una cultura de respeto de los derechos de la niñez migrante" (Fundación Anide, s. f.). Dicho colectivo, contaba a la fecha de la experiencia con un equipo multiprofesional de profesionales y estudiantes en práctica dedicados al trabajo con las niñas, niños y adolescentes, la articulación con otras organizaciones y con el Estado, la asesoría a familias migrantes y las tareas administrativas. El trabajo dirigido a las niñas, niños y adolescentes consistía, para entonces, principalmente en apoyo escolar, realización de talleres, apoyo psicológico y de asistencia social. Resulta relevante ofrecer un análisis sobre dicha experiencia, porque corresponde a una propuesta de acción fonoaudiológica en un espacio no gubernamental, en que las prácticas biomédicas podían cuestionarse y desarrollar un trabajo horizontal para la construcción de conocimiento colectivo. 
Los participantes de la experiencia se pueden dividir en dos grupos:

- El grupo externo: compuesto por cinco estudiantes de cuarto año de la carrera de Fonoaudiología de la Universidad de Chile.

- El grupo interno: compuesto por aproximadamente 20 niñas, niños y adolescentes, de entre 6 y 14 años, que asistían al Colectivo de lunes a viernes, después de su jornada escolar regular. Dentro del Colectivo, las niñas, niños y adolescentes realizaban sus tareas escolares, de manera individual y guiada por las y los tutores del apoyo escolar, además de participar en talleres u otras actividades que se programaran.

La propuesta del grupo de estudiantes de Fonoaudiología consistió en un proyecto de investigación acción participativa en el interior del Colectivo, basados en la convicción de generar conocimiento fuera del contexto clínico biomédico tradicional. Dentro del Colectivo, las acciones se realizaron con las niñas, los niños y jóvenes que asistían a dicho espacio después de su jornada escolar (Calixto et al., 2013). Tal como se describirá en detalle más adelante, la experiencia consistió, grosso modo, en la participación dentro del acompañamiento escolar y en la posterior realización de acciones participativas, bajo los principios de una Investigación Acción Participante (Fals-Borda, 2009; Krause, 2002).

El registro de las experiencias, emociones y análisis personales se plasmó en bitácoras personales, las cuales se compartían y analizaban en reuniones periódicas con los tutores de la investigación y cuyos extractos seleccionados constituyen la base de la tesis de Calixto et al. (2013).

\section{ANÁLISIS DE LA EXPERIENCIA}

El análisis de la experiencia se realizó a partir de la selección de extractos de las bitácoras de campo del equipo de estudiantes participantes publicadas en la tesis de grado mencionada. Dicho análisis se llevó a cabo en base a los principios y dimensiones del paradigma de la construcción y transformación crítica de (Montero, 2004) caracterizadas en el cuadro 1. Los autores de este informe procedieron a identificar e interpretar las características de la experiencia a partir de los elementos constitutivos de cada dimensión propuesta por Montero. Para ello se realizó un ejercicio interpretativo flexible, de inspiración holística y relacional, que alternó múltiples instancias recursivas de análisis individual, discusión y alcance de consensos interpretativos y analíticos grupales. Los extractos de las bitácoras se analizaron con distintas herramientas, por ejemplo, con tablas de matrices que contenían, por un lado, las cinco dimensiones del paradigma y, por otro, la temporalidad de las acciones realizadas. Todo este análisis permite proponer el reconocimiento de tres momentos en el transcurso de la experiencia, los cuales en su conjunto dan cuenta del alto grado de complejidad y dinamismo del proceso. A continuación, se describen estos tres momentos observados.

\section{Momento 1: Inicio de la experiencia}

La participación comenzó con la inclusión del equipo de estudiantes como tutores en el espacio de apoyo escolar, realizado tres días de la semana, por dos horas. Esta instancia tenía por objetivo acompañar a las niñas, niños y jóvenes en la realización de tareas escolares y facilitar estrategias de aprendizaje. Para el equipo de estudiantes, las sesiones de apoyo escolar significaron un primer acercamiento al funcionamiento del centro y a las dinámicas relacionales entre los distintos actores (funcionarios, voluntarios, padres y madres, niños, niñas y jóvenes).

Durante los primeros meses, los estudiantes de Fonoaudiología experimentaron un profundo proceso de ajuste, tanto de identidad grupal para interactuar con los otros integrantes de la institución, como de regulación de expectativas sobre su rol en dicho espacio. Se enfrentaron, por ejemplo, a las prácticas de interacción entre los niños, niñas y jóvenes, quienes se organizaban autónomamente para decidir el lugar del colectivo en el cual trabajar, el tipo de tarea que realizar y armar grupos por afinidad.

“(...) En el momento de comenzar con el apoyo escolar en concreto, los niños se sentaron y llevaban sus cuadernos, sabiendo cómo era la forma de actuar. Me fue muy significativo observar el desplante con el que se movilizaban en el Colectivo, reconociéndolo como espacio propio y con nula incomodidad".

En este momento, los estudiantes de Fonoaudiología evidenciaron sensaciones de incomodidad al enfrentarse a estas dinámicas relacionales, percibieron limitaciones en su actuación dentro del espacio, y frustración al advertir una falta de preparación para trabajar con grupos, a pesar de la motivación inicial de generar un trabajo en una organización social, desde una perspectiva no individual.

"Debo admitir que experimenté muchos sentimientos, entre la frustración por no obtener que los niños entendieran, desesperación por mi carencia de recursos pedagógicos y enojo por sentirme ignorado por los niños." 
Esta vivencia se plasmó en la posición que el equipo de trabajo adoptó en sus primeras semanas de interacción, influenciada por el saber profesional biomédico y normalizador de su formación, en algunas representaciones sociales respecto al trabajo comunitario con niños y niñas migrantes, marcadas por estigmas asociados al estatus migratorio y una mirada paternalista sobre la niñez. Desde esta perspectiva, las prácticas de socialización de los niños, niñas y jóvenes se volvieron difíciles de comprender, pues iban alternando interacciones de apoyo con otras de rechazo o violencia. Esta situación generó la emergencia de juicios sobre los niños, niñas y jóvenes, con foco en las prácticas disruptivas, las dificultades en el plano académico y aspectos patologizantes.

"La dinámica que se da entre los niños es bastante hostil, no hay una relación de compañerismo, ayuda mutua, solidaridad en el momento. Se deben fortalecer los patrones relacionales $y$ de interacción social que promuevan el respeto, la solidaridad y el trabajo en equipo."

“(...) Me llamó inmediatamente la atención su interposición lingual al hablar; fue inevitable comenzar de a generar hipótesis de por qué lo tenía. ¿MHO? [Malos Hábitos Orales] ¿debilidad lingual? me dio hasta risa pensar en eso tan compulsivamente, supongo que no es tan fácil despojarse de ese lado patologista."

Esta visión paternalista del rol profesional los llevó, en un primer momento, a visualizar su actuación desde la intervención, al desear modificar situaciones que consideraban deficitarias desde sus parámetros, tanto en la organización de los espacios y lineamientos del apoyo escolar, como en las habilidades cognitivas de los niños, niñas y jóvenes, lo que tensionó al equipo y la relación con los integrantes del Colectivo. En este escenario, había imposibilidad de observar el despliegue de un conjunto de acciones y recursos por parte de los niños y niñas, y de reconocer en ellas y ellos a sujetos de derecho, autónomos para tomar decisiones.

\footnotetext{
"Admito que me exaspera bastante el típico comentario de "tú no eres la que manda, tú solo nos ayudas en las tareas así que no me importa". Me rehúso a pensar que la única manera de controlar ese tipo de cosas es poniéndome seria y con una actitud más autoritaria...tiene que haber otra forma, pero lo que sé es que tanto esa opción como el no hacer nada me parecen desgastantes."
}

Con el transcurso del tiempo, el afianzamiento de las relaciones entre los niñas, niños, jóvenes e integrantes del equipo de Fonoaudiología, junto a un proceso reflexivo de sus prácticas, permitió generar una reinterpretación de las dinámicas de interacción, comprender los elementos estructurales que configuran las relaciones entre ellos y ampliar el espectro de posibilidades de vinculación, más allá de las acciones motivadas por un fin académico. En paralelo, la identidad grupal de los estudiantes también se transformó, al reconocerse con necesidades, emociones e intereses en común dentro del espacio. Estos cambios, no lineales, permiten ir reconociendo un segundo momento en la experiencia.

\section{Momento 2: Transición}

Uno de los procesos que marcó un punto de inflexión en el tránsito hacia trabajar bajo un modelo comunitario propiamente tal es la modificación de las relaciones establecidas entre el equipo y los niños, niñas y jóvenes del Colectivo Sin Fronteras. Durante el proceso de reconocimiento recíproco, se gestó un vínculo que se fue caracterizando por la valoración de los intereses de las distintas personas, la validación de las emociones y la negociación de roles entre los integrantes del apoyo escolar. Este hecho, propició espacios de encuentro y diálogo para compartir experiencias de vida, sentimientos y deseos.

"Z. me hizo muchas preguntas mientras pintábamos (si tenía hermanas, qué estudio, mi edad, donde vivo, si tengo pololo). Me hacía un poco de gracia que cada vez que me preguntaba algo hacía ademán de arrepentirse, como si hubiera estado mal preguntarme (...) Yo también aproveché de preguntarle cosas, y entre ellas me comentó que era de Perú, le gustaba ir al colegio (cosa rara) y que le gustaba mucho religión, inglés y música. Conversamos sobre lo que significaba lo que estábamos pintando, y me puse a contarle un poco la historia."

En la medida que este vínculo se fortalecía, aumentó la disposición a trabajar de manera colaborativa y se desdibujó la visión de ser un experto externo, para pasar a sentirse parte del espacio. Los anteriores juicios estigmatizadores se transformaron en reflexiones situadas $\mathrm{e}$ intercambios de visiones $\mathrm{y}$ comprensiones de las actuaciones de acuerdo con la historia de vida de cada sujeto.

"Es cierto que se exige mucha atención y sensibilidad a todo lo que esté ocurriendo, pero me sentí muchísimo mejor, cuando decidi salir del rol externo, y decidir aceptar lo que esta comunidad de niños y niñas, finalmente, me ofrecia: ser parte de su comunidad, por lo menos dentro del espacio."

En este punto, surge por parte de los estudiantes la voluntad de propiciar espacios de participación de los niños, niñas y jóvenes desde un enfoque de derechos, pues se consideró restringido el 
formato del apoyo escolar para este fin. Esto motivó reuniones con los integrantes del Colectivo Sin Fronteras, que implicaron conocer la necesidad que dio origen al apoyo escolar, reconstruir su desarrollo a través del tiempo y reflexionar sobre las condiciones actuales del espacio. A raíz de esto, se planificó un diagnóstico participativo, según las propuestas de la educación popular, el cual tuvo como objetivos: a) valorar las fortalezas y debilidades del apoyo escolar por parte de los participantes del espacio, b) planificar junto con los niños, niñas y jóvenes un segundo espacio de interacción, el taller Aprender Sin Cuadernos. Este diagnóstico participativo se realizó el 11 de julio de 2013, contó con la participación de las niñas, niños y adolescentes y del grupo de estudiantes de Fonoaudiología y se desarrolló sobre la base de dos planteamientos para discutir: 1. Lo que me gusta del apoyo escolar y 2. Lo que mejoraría del apoyo escolar. Se observa en este punto que la necesidad de la evaluación es instalada de manera unilateral por el grupo de estudiantes; aun así, el grupo de niñas, niños lo subvirtieron para direccionarlo en el sentido de sus legítimos intereses y preocupaciones.

"A muchos les gustaría tener actividades de juego. Nosotros les prometimos realizar esto último antes del apoyo. Se viene una gran demanda entonces, ya que la idea será realizar juegos de colaboración y de equipo entre todos."

Como se observa en este fragmento, existió una suerte de desconcierto positivo en el grupo de estudiantes frente a la emergencia de las niñas, los niños y adolescentes como sujetos con voz propia sobre su realidad, que muestra cierta tensión entre el modelo participativo y el control de las decisiones por parte de los adultos.

Este proceso de ajuste y transformación permitió que el grupo de estudiantes se abriera a la generación de vínculos emocionales con las niñas, niños y adolescentes $\mathrm{y}$, por lo tanto, comenzara a forjarse una relación nueva entre ellos. Ese proceso conllevó un nuevo y último replanteamiento de la finalidad de la presencia de ellas y ellos en ese espacio, lo que permitió que emergiera la posibilidad de construirse desde el rol de acompañantes o facilitadores de un proceso colectivo.

\section{Momento 3: Reconfiguración del trabajo}

A partir de la información emanada en el diagnóstico participativo, se implementaron las modificaciones en el apoyo escolar. Al inicio de cada jornada, se implementó un espacio de juegos de 20 minutos, en el que se buscó potenciar el enfoque de derechos que guiaba el funcionamiento del Colectivo. Dentro de los elementos abordados en los juegos se encontraron: el trabajo en equipo, el respecto por el otro, la honestidad, las habilidades para favorecer el diálogo, entre otros. Prontamente, los niños y niñas se apropiaron de esta nueva rutina, aprendieron a defenderla $\mathrm{y}$ adquirieron mayor protagonismo en las decisiones de las dinámicas lúdicas a través de su participación en la planificación de los juegos. Para tal caso, el equipo incorporó a los niños y niñas en la organización de las rutinas dentro del colectivo. En este punto, se advierte un cambio de mirada de los estudiantes hacia los niños y niñas y una valoración de la reciprocidad agentiva de dichos cambios.

De manera paralela, se llevó a cabo el taller Aprender Sin Cuadernos, instancia que buscaba potenciar competencias en los niños y niñas para el abordaje de situaciones complejas. Dada la demanda de los padres, madres y tutores por mejorar el rendimiento académico de los niños y niñas, junto con la necesidad de estos últimos por contar con espacios de expresión y convivencia, el equipo decidió posicionar al discurso como eje central de la propuesta para, por un lado, favorecer el desarrollo de habilidades comunicativas para el desempeño académico y, por otro, fortalecer la comunicación como una herramienta para el ejercicio de los derechos de la niñez y cohesión social. Es decir, a partir de este encuadre, las acciones se enmarcaron en una perspectiva promocional del desarrollo humano. Lamentablemente, el proceso se vio interrumpido en el mes de noviembre, al finalizar el año académico, lo que impidió que el proceso en espiral previsto en un proyecto de investigación acción participativa se completara totalmente.

Con esto, sin embargo, se observa que el equipo pasó de una identidad interventora, centrada en los aspectos negativos del escenario y los actores, a la comprensión de un rol facilitador de un proceso de fortalecimiento de grupo y promoción de derechos; de un enfoque centrado en el déficit a uno promotor de habilidades. Además, se observa cómo fue desarrollándose, a lo largo de toda la experiencia, una relación entre estudiantes, niños, niñas y adolescentes que, con tensiones y complejidades, terminó afiatada en un trabajo colectivo y propositivo. Este tercer momento finalizó en noviembre de ese año, con el término del año académico. El grupo de estudiantes prosiguió su participación en el Colectivo por un tiempo más, pero el registro de dicha participación se interrumpió, por la necesidad de sistematizar la investigación en un escrito.

\section{Valoración de la experiencia desde las dimensiones}

En la descripción ofrecida del proceso de trabajo con las niñas, niños y adolescentes del Colectivo Sin Fronteras, se ha recurrido, de manera no explícita, a algunos elementos definitorios de las dimensiones ontológica, epistemológica, metodológica, ética y 
política que Montero propone para enmarcar una experiencia en el paradigma comunitario. A continuación, se ofrece una interpretación explícita de encuadre de la experiencia desde estas cinco dimensiones.

En lo que hemos identificado como primer momento, puede observarse el establecimiento de dinámicas relacionales entre los sujetos bajo formas de actuación y representación muy distantes al enfoque comunitario. Es claro, por ejemplo, que la mirada que los distintos protagonistas tienen tanto de sí mismo como de los otros no se construye en cuanto actores, en el sentido ontológico, es decir, como sujetos activos y autónomos. Por una parte, las niñas, los niños y adolescentes conforman un grupo heterogéneo, con el cual se privilegia un trabajo individualizado y sobre el cual los adultos ejercen sus decisiones; por otra parte, las y los estudiantes de Fonoaudiología irrumpen en el espacio del Colectivo con la conciencia de prácticas académica que desean subvertir, pero que igualmente ponen en acción, al primar una visión interventora, con el deseo de romper con la lógica biomédica formativa, pero sin claridad aún sobre los recursos para reemplazar esa lógica por otra. Esto marca que, inicialmente, el conocimiento se conciba como perteneciente a un solo grupo, el de los estudiantes de Fonoaudiología, quienes buscan la generación unilateral de diagnósticos, con una mirada centrada en el déficit y en un determinismo social. En ese sentido, las niñas, niños y adolescentes no son visualizados como sujetos que conforman un colectivo, sino como objetos de observación, intervención y compasión, por lo que prima sobre ellos una visión de paternalismo tradicional (Liebel, 2007). El espacio de interacción, el apoyo escolar, es un espacio no motivado ni propuesto por ninguno de los dos grupos en vinculación, por lo tanto, es un espacio poco propicio para la transformación y la generación colectiva de conocimiento. En ese espacio, la relación es tensa, con numerosos ejemplos de no reconocimiento mutuo, en que muy probablemente para ninguna de las dos partes existe un otro como sujeto propiamente tal. En este escenario, es evidente que resultaba imposible visualizar un horizonte transformador, una praxis con "función desalienante, movilizadora de la conciencia y socializadora" (Montero, 2004), pese a que existe la intención de moverse, de alguna u otra manera, en esa dirección.

En el segundo momento descrito puede observase la vivencia de la crisis que implica la toma de conciencia de ese primer momento. Es, en términos éticos, el momento del reconocimiento. Las niñas, niños, adolescentes y el equipo de estudiantes comienzan, poco a poco, a establecer vínculos afectivos, lo que fractura la mutua percepción inicial de seres objetos. La visión deficitaria sobre los niños va cambiándose paulatinamente por una en que comienzan a reconocerse sus habilidades. Es decir, aún no se logra percibir a las niñas, niños y adolescentes como sujetos plenamente activos que construyen su realidad en interacción con las condiciones históricas y sociales que viven, pero se abandona el paternalismo tradicional. En paralelo, las y los estudiantes comienzan a redefinir su identidad profesional. Esta etapa está marcada por procesos relacionales y epistemológicos ambivalentes: se visualiza la distancia que hay entre la acción concurrente y los principios del enfoque comunitario, a la vez que comienza un redireccionamiento de la experiencia, en términos de su finalidad. En ese marco, surge la ideación y realización de los diagnósticos participativos con las niñas, niños y adolescentes.

En el tercer momento identificado, el equipo de Fonoaudiología se visualiza como observadores participantes o facilitadores. Se visualiza a las niñas, niños y adolescentes como sujetos de derechos, con el esfuerzo constante de reconocerlos como personas activas, con voz, con recursos y necesidades, más allá de lo académico, que conforman un colectivo y con quienes se establecen vínculos afectivos y de conocimiento mutuo. La voz de ellos, sin embargo, si bien es catalizadora de procesos, no logra adquirir toda la autonomía propia de procesos comunitarios, pues la decisión del curso de acciones sigue estando bajo el control de los adultos. En ese sentido, podría señalarse que la visión hacia las niñas, niños y adolescentes se mueve hacia un paternalismo moderno (Liebel, 2007). Sin embargo, el trabajo en conjunto se direcciona hacia el conocimiento y reflexión de los derechos de la niñez, se visualiza un potente foco de transformación en sus relaciones presentes y futuras, y se enriquece la participación de los niños y niñas, especialmente en términos colectivos.

Además, puede observarse que en esta etapa existe un acercamiento a la manera en que Montero caracteriza al actor profesional comunitario, especialmente en términos de una función de colaboración, intercambio creciente de saberes, una profunda redefinición de roles convencionales, con un foco en la identificación de potencialidades, el fortalecimiento y la puesta en prácticas de estas. También, debe señalarse que se observa una redefinición del ámbito de actuación fonoaudiológica, desde un momento inicial en que este se centraba en la comunicación humana en términos de déficit lingüístico y dificultades interaccionales, hacia un momento final en que se observa tanto como recurso por potenciar, como medio catalizador para abordar los derechos. Ambas perspectivas de trabajo transforman de manera radical la visión fonoaudiológica biomédica sobre la comunicación y la provee de un fuerte componente ético y político, como recurso basal para procesos de autonomía, en este caso, de un colectivo de niñas y niños migrantes y sus familias. 
Por último, se observa que, desde la dimensión metodológica, la experiencia fue incompleta. El proceso participativo se concibe como un proceso en espiral, en que se secuencia instancias de planificación, acción colectiva, observación sistemática, reflexión colectiva y replanificación (Fals-Borda, 2009; Krause, 2002). Dicho proceso en espiral es evidentemente interrumpido en la etapa de la acción colectiva y observación sistemática, sin lograr retomar instancias de reflexión y replanificación, que generaran el sentido de espiral en la generación de conocimiento colectivo. El escenario académico de la experiencia marcó en gran medida esta incompletitud metodológica, lo que permite poner un foco sobre las complejas relaciones entre instituciones académicas y colectivos.

En definitiva, el análisis de la experiencia desde las cinco dimensiones señaladas, permiten identificar los aspectos más fortalecidos y débiles a la luz de un paradigma comunitario. En términos ontológicos, se instala un reconocimiento mutuo de actores sociales, aunque la representación de las niñas, niños y adolescentes como sujetos plenos queda a medio camino. El conocimiento se genera en la interacción, de manera dinámica, lo que permite superar intervenciones desde el déficit y el paternalismo e instalar en el centro de la acción y de la reflexión los recursos comunicativos puestos en la interacción y la relación. La implementación metodológica va en el sentido de instalar la participación comprometida de los distintos actores, pero la necesidad misma del proceso es decidida unilateralmente y el proceso queda interrumpido antes de una primera evaluación comunitaria de sus resultados, lo que impide plasmar la lógica de espiral en la construcción del conocimiento propia del modelo. En términos éticos, se establece una relación entre sujetos que crecientemente se reconocen como tales, con dinámicas propias, transformadoras de las identidades y de las prácticas comunicativas y relacionales. Por último, se reconoce de manera creciente la conciencia sobre la función transformadora de la relación y la comunicación, especialmente en término de derechos y de autocuidado colectivo.

\section{DISCUSIÓN FINAL}

En este artículo se propuso evaluar una experiencia planteada desde los supuestos de una investigación acción participativa en una organización social, llevada a cabo por un equipo de estudiantes de Fonoaudiología, a la luz de las características que Montero (2004) propone para definir el paradigma comunitario. Esta evaluación no se propone por un afán academicista, sino para relevar la complejidad inherente $y$, a la vez, ineludible de abordar la transformación del enmarque biomédico, asistencialista, ahistórico e individual que prima actualmente en la Fonoaudiología, lo que limita radicalmente sus posibilidades de estar al servicio de procesos transformadores. En el ámbito latinoamericano se ha desarrollado el abordaje comunitario como un posible camino para aportar decididamente en los procesos emancipadores de los grupos vulnerados, pero ese abordaje debe realizarse en observación de sus principios configuradores, en que la actuación profesional está al servicio del trabajo y escrutinio colectivo, y en el que la generación de conocimiento se concibe como un proceso complejo, dinámico, que implica desafiar supuestos esenciales de las lógicas académicas hegemónicas, propias de la modernidad imperante (Flores, 2012; Harding, 1996; Rozas, 2015; Santos, 2011).

¿Cuál es el sentido de lo comunicativo para una comunidad migrante, en el contexto de un país con una ley migratoria que no garantiza derechos sociales y de una sociedad contradictoria en sus prácticas de acogida y de discriminación? ¿Cuál es sentido de la promoción de derechos con una comunidad de niñas, niños y adolescentes, sobre quienes pesan prácticas de paternalismo? La Fonoaudiología actualmente se concibe como un ámbito académico y profesional en que priman las características modernas y coloniales, materializadas en el modelo biomédico hegemónico, por lo que las respuestas no son fáciles. Si la Fonoaudiología realmente quiere aportar con sentido transformador en contextos como este y otros de vulnerabilidad, debe afrontar con decisión, humildad y seriedad interrogantes como estas, y confrontar críticamente las bases positivistas y colonizantes sobre las que se funda, al igual que las demás profesiones de la salud (García-Ruiz, 2014; Martínez Hernáez, 2011; Ossandón, 2014; Soto, 2014).

El ámbito comunitario no consiste en sumar un área más a las disciplinas y profesionales, sino en transformarlas ante la comprensión de que el ser humano es un ser relacional. Pensar una Fonoaudiología comunitaria como un espacio más entre otros solo sería reforzar la definición positivista de "objetos" específicos de estudio (González, 2014). En la línea señalada por Silva (2009) y Alfaro (2000), se puede aspirar a dar, desde lo comunicativo y el desarrollo humano, un salto cualitativo desde una conciencia ingenua hacia una conciencia crítica y a participar de acciones profesionales que aporten al bienestar de la población, y eliminar las injusticias sociales. El ámbito profesional sobre el que se funda la Fonoaudiología, la comunicación humana y su desarrollo, ofrece todo el potencial para contribuir a personas y colectivos en procesos de transformación social. 


\section{REFERENCIAS}

Alfaro, J. (2000). Discusiones en Psicología Comunitaria (R. Morales, Ed.). Universidad Diego Portales. http://www.psicosocial.net/historico/index.php?option=com_docman\&view=do wnload\&alias $=558$-discusiones-en-psicologia-

comunitaria\&category_slug=psicologia-comunitaria\&Itemid=100225

Calixto, B., Jaramillo, P., Larenas, D., Martínez, F., \& Muñoz, N. (2013). Proposiciones desde la fonoaudiología para la intervención psicosocial con enfoque comunitario: Una experiencia dirigida a niños emigrantes [Seminario de Licenciatura, Universidad de Chile]. http://repositorio.uchile.cl/handle/2250/116749

Cohen, H., \& Natella, G. (2013). La Desmanicomialización: Crónica de la reforma del Sistema de Salud Mental en Río Negro. Lugar Editorial.

Escobar, A., Kothari, A., Acosta, A., Demaria, F., \& Salleh, A. (2020). Encontrando senderos pluriversales. En L. L. North, B. Rubio, \& A. Acosta (Eds.), Concentración económica y poder político en América Latina. Fundación Friedrich Ebert, Representación en México; CLACSO. http://biblioteca.clacso.edu.ar/clacso/se/20200319051943/Concentracioneconomica.pdf

Fals-Borda, O. (2009). Una sociología sentipensante para América Latina ( $\left.1^{\mathrm{a}} \mathrm{ed}.\right)$. Siglo del Hombre; CLACSO. http://bibliotecavirtual.clacso.org.ar/ar/libros/coedicion/fborda/fborda.pdf

Flores, J. M. (2012). Psicología Comunitaria, Opresión y Exclusión. / Community Psychology, Oppression and Exclusion. Liminales. Escritos sobre psicología y sociedad, $\quad l(2), \quad 117-136$. http://revistafacso.ucentral.cl/index.php/liminales/article/view/226

Fundación Anide. (s. f.). Colectivo Sin Fronteras. Colectivo Sin Fronteras. Recuperado 13 de agosto de 2020, de http://anide.cl/programa2proyecto2.php

García-Ruiz, S. (2014). La RBC donde la pobreza, la riqueza y el desarrollo local se encuentran. En A. Guajardo, E. Recabarren, \& V. Parraguez (Eds.), Rehabilitación de base comunitaria. Diálogo, reflexiones y prácticas en Chile (pp. 20-29). Universidad Andrés Bello. Servicio Nacional de Discapacidad. https://issuu.com/unab/docs/rbc_en_chile

García-Ruiz, S., Vásquez, A., \& Guajardo, A. (2019). Rehabilitación Basada en la Comunidad: Una estrategia local para el acceso a derechos y el ejercicio de la ciudadanía. Editorial Universidad de Santiago de Chile. https://www.fundacionideal.org.co/sites/default/files/imce/libro_rbc_2019.pdf

González, F. (2014). Dilemas epistemológicos actuales en psicología comunitaria. En J. Flores (Ed.), Repensar la psicología y lo comunitario en América Latina. Universidad de Tijuana. http://www.comunidadeslatinoamericanas.uchile.cl/publicaciones/revistas/cic/re pensar_psicologia_y_comunitario.pdf

Harding, S. (1996). Ciencia y feminismo. Ediciones Morata. https://edmorata.es/libros/ciencia-y-feminismo/

Krause, M. (2001). Hacia una redefinición del concepto de comunidad -cuatro ejes para un análisis crítico y una propuesta-. Revista de Psicología, 10(2), 49-60. https://doi.org/10.5354/0719-0581.2001.18572

Krause, M. (2002). Investigación-acción-participativa: Una metodología para el desarrollo de autoayuda, participación y empoderamiento. En J. W. Durston \& F. Miranda (Eds.), Experiencias y metodología de la investigación participativa.
CEPAL, División de Desarrollo Social.

Liebel, M. (2007). Paternalismo, participación y protagonismo infantil. En Y. Caraveo \& M. E. L. Pontón (Eds.), Participación infantil y juvenil en América Latina. Universidad Autónoma Metropolitana. http://www.childwatchlac.org/participacion-infantil-y-juvenil-en-america-latina/

Marconi, J. (1972). Asistencia psiquiátrica intracomunitaria en el área sur de Santiago. Bases teóricas y operativas para su implementación (1968-1970). Revista Chilena de Neuro-Psiquiatría, 11(2), 3-12.

Martínez Hernáez, Á. (2011). La copia de los hechos: La biomedicina, el poder y sus encubrimientos. Quaderns, 27, 45-64. https://dialnet.unirioja.es/servlet/articulo? codigo $=3958789$

Martínez, M. (2006). El enfoque comunitario. Estudio de sus modelos de base. Universidad de Chile, Facultad de Ciencias Sociales. http://repositorio.uchile.cl/handle/2250/122235

Montero, M. (2004). Introducción a la psicología comunitaria: Desarrollo, conceptos $\quad y \quad$ procesos. Paidós http://www.psicosocial.net/historico/index.php?option=com docman\&view=do wnload\&alias=737-introduccion-a-la-psicologia-comunitaria-desarrolloconceptos-y-procesos\&category_slug=psicologia-comunitaria\&Itemid=100225

Ossandón, M. P. (2014). Rehabilitación basada en la comunidad frente a la realidad chilena. Revista Chilena de Terapia Ocupacional, 14(2), 219-230. https://doi.org/10.5354/0719-5346.2014.35724

Prilleltensky, I. (2004). Prólogo. Validez psicopolítica: El próximo reto para psicología comunitaria. En M. Montero (Ed.), Introducción a la psicología comunitaria: Desarrollo, conceptos y procesos. Paidós. http://www.psicosocial.net/historico/index.php?option=com_docman\&view=do wnload\&alias=737-introduccion-a-la-psicologia-comunitaria-desarrolloconceptos-y-procesos\&category_slug=psicologia-comunitaria\&Itemid=100225

Rozas, G. (2015). Hacia una Psicología Social Comunitaria del Sur. Psicología $\begin{array}{lllll}\text { Conocimiento } & y & \text { Sociedad, 278-306. }\end{array}$ https://revista.psico.edu.uy/index.php/revpsicologia/article/view/265

Santos, B. de S. (2011). Epistemologías del sur. Utopía y Praxis Latinoamericana, 16(54), 17-39. http://www.boaventuradesousasantos.pt/media/EpistemologiasDelSur Utopia\%2 0y\%20Praxis\%20Latinoamericana_2011.pdf

Silva, A., Escudero, P., Hidalgo, R., \& del Campo, M. (2018). Estudio Cualitativo de la Práctica Fonoaudiológica en el Contexto de la Atención Primaria de la Salud, en la Quinta Región de Valparaíso. Ciencia \& Trabajo, 20(62), 103-106. https://doi.org/10.4067/S0718-24492018000200103

Silva, J. (2009). Medicina comunitaria: Introducción a un análisis crítico. Salud Colectiva, 5(1), 121-126. https://doi.org/10.18294/sc.2009.234

Soto, H. (2014). Implementación de la estrategia de Rehabilitación Basada en Comunidad: Una reflexión participante. En A. Guajardo, E. Recabarren, \& V. Parraguez (Eds.), Rehabilitación de base comunitaria. Diálogo, reflexiones y prácticas en Chile (pp. 20-29). Universidad Andrés Bello. Servicio Nacional de Discapacidad. https://issuu.com/unab/docs/rbc_en_chile

Tapia, S., Espinoza, F., Herrera, P., \& Venegas, D. (2016). Caracterización de fonoaudiólogos/as insertos/as en Centros Comunitarios de Rehabilitación. Revista Chilena de Fonoaudiología, 15, 1-13. https://doi.org/10.5354/rcdf.v15i0.44186 